\title{
DISTRIBUTIVITY AND PERMUTABILITY OF CONGRUENCE RELATIONS IN EQUATIONAL CLASSES OF ALGEBRAS
}

\author{
A. F. PIXLEY
}

A well known theorem of Malcev [1] (Theorem 1, below) provides a characterization of those equational classes of algebras whose members have permutable congruence relations. The present note presents a companion to this theorem dealing with equational classes whose members possess distributive lattices of congruence relations.

1. Preliminary concepts. In slightly different terminology and notation, all of the concepts and results discussed in this section (except Theorem 1) are to be found in the work of Birkhoff $[2 ; 3]$.

Let $T$ be an arbitrary, fixed, index set and let $S$ be a function on $T$ to the set of positive integers. $\mathfrak{A}$ is an algebra of species $S$ if $\mathfrak{A}$ is a mathematical system $\mathfrak{U}=(A, K)$ consisting of a nonempty set $A$ and a family $K$ of primitive operations $o_{\alpha}, \alpha \in T$, where $o_{\alpha} \in A^{A^{S(\alpha)}}$. We denote by $o_{\alpha}$ the $S(\alpha)$-ary operation of any algebra of species $S$, observing that $o_{\alpha}$ will have a different interpretation in different algebras. With this in mind we have the following definitions.

An $S$-expression is either an indeterminate or a finite composition of indeterminates with the $o_{\alpha}$.

An $S$-identity is an equation $\phi=\psi$ where $\phi$ and $\psi$ are $S$-expressions.

An algebra $\mathfrak{A}$ of species $S$ satisfies the $S$-identity $\phi=\psi$ if for each (single valued) assignment of elements of $A$ to the indeterminates appearing in $\phi$ or $\psi, \phi$ yields the same member of $A$ as $\psi$.

Let $U$ be any set of $S$-identities. By $C(U)$ we denote the equational class of species $S$ satisfying $U$ consisting of those algebras of species $S$ which satisfy all of the $\phi=\psi \in U$. For example, if $\cup$ and $\cap$ are taken as the primitive operations, and $U$ consists of the identities:

(i) $x \cup y=y \cup x, x \cap y=y \cap x$,

(ii) $x \cup(y \cup z)=(x \cup y) \cup z, x \cap(y \cap z)=(x \cap y) \cap z$,

(iii) $x \cup(x \cap y)=x \cap(x \cup y)=x$,

then $C(U)$ consists of the class of all lattices.

If $k$ is any cardinal number and $U$ is any set of $S$-identities, we denote by $\mathfrak{F}_{k}(U)$ the free algebra of species $S$, having $k$ generators, determined by the identities $U$. $\mathfrak{F}_{k}(U)$ has as elements classes of $S$ expressions in $k$ indeterminates where two expressions $\phi$ and $\psi$ are placed in the same class if $\phi=\psi$ is a logical consequence of the identities $U$. It can be shown [2], and in the sequel we require the fact, that $\mathfrak{F}_{k}(U) \in C(U)$.

Received by the editors October 6, 1961 and, in revised form, December 23, 1961. 
Let $\theta_{1}$ and $\theta_{2}$ be congruence relations on an algebra and let $\theta_{1} \circ \theta_{2}$ denote their relative product. If $\theta_{1} \circ \theta_{2}=\theta_{2} \circ \theta_{1}$ then $\theta_{1}$ and $\theta_{2}$ are said to be permutable. Permutable congruence relations are discussed in [3] from which we recall: The congruence relations on any algebra with permutable congruence relations form a modular lattice in which $\theta_{1} \cup \theta_{2}$ $=\theta_{1} \circ \theta_{2}=\theta_{2} \circ \theta_{1}$.

Distributivity of the lattice of congruence relations of an algebra is another condition which, like permutability, implies modularity. The importance of permutability, distributivity, and modularity of congruence relations for the direct and subdirect sum representation and uniqueness theorems of algebra is discussed in Chapter VI of [3].

If $U$ is any set of $S$-identities and if each $\mathfrak{A} \in C(U)$ has permutable congruence relations, we say that $U$ is a permutable set of identities. If each $\mathfrak{A} \in C(U)$ has a distributive lattice of congruence relations, we say that $U$ is a distributive set of identities.

In terms of these conventions the theorem of Malcev, referred to above, may be stated as follows:

Theorem 1. Let $C(U)$ be an equational class of species $S$. The following two conditions are equivalent:

(i) There is an $S$-expression $\pi(x, y, z)$ such that each of $\pi(x, x, z)=z$ and $\pi(x, z, z)=x$ is a logical consequence of the identities $U$.

(ii) $U$ is a permutable set of identities.

For example, let $C(G)$ be the equational class of groups with operations $\times$ and $^{-1}$, taking $G$ as the set of identities:
(i) $x(y z)=(x y) z$,
(iii) $x x^{-1}=y y^{-1}$,
(ii) $x x^{-1}=x^{-1} x$,
(iv) $x\left(y y^{-1}\right)=x=\left(y y^{-1}\right) x$.

Then, taking $\pi(x, y, z)=x y^{-1} z, G$ is seen to be a permutable set.

2. Equational classes with distributive lattices. The purpose of the present note is to establish:

Theorem 2. Let $C(U)$ be an equational class of species $S$. If

(i) there is an S-expression $\sigma(x, y, z)$ such that each of $\sigma(x, x, z)=x$, $\sigma(x, y, x)=x$, and $\sigma(x, z, z)=z$ is a logical consequence of the identities $U$, then

(ii) $U$ is a distributive set of identities and the infinite distributive law $\theta \cap \cup \theta_{i}=U\left(\theta \cap \theta_{i}\right)$ is satisfied by the congruence relations of the members of $C(U)$.

The identities $U$ are both permutable and distributive if and only if both (i) above and (i) of Theorem 1 are satisfied.

Proof. Suppose that condition (i) holds and that $\mathfrak{A} \in C(U)$. Let 
$\theta$ and $\theta_{i}, i \in P, P$ an arbitrary index set, be any congruence relations of $\mathfrak{A}$. Since

$$
\theta \cap U \theta_{i} \geqq U\left(\theta \cap \theta_{i}\right)
$$

in any lattice, and since each of the dual finite distributive laws always implies the other, to establish (ii) we need only show that

$$
\theta \cap \bigcup_{i \in P} \theta_{i} \leqq \bigcup_{i \in P}\left(\theta \cap \theta_{i}\right) \text {. }
$$

Suppose then, that $a, b \in A$ and

$$
a \equiv b(\theta), \quad \text { and } \quad a \equiv b\left(\bigcup_{i \in P} \theta_{i}\right)
$$

Then there is a finite sequence $a=x_{0}, x_{1}, \cdots, x_{n}=b$ of elements of $A$ such that

$$
x_{j-1} \equiv x_{j}\left(\theta_{i_{j}}\right) \text { for some } i_{j} \in P, j=1, \cdots, n .
$$

We exhibit a finite sequence $a=y_{0}, y_{1}, \cdots, y_{n}=b$ of elements of $A$ such that

$$
y_{j-1} \equiv y_{j}\left(\theta \cap \theta_{i_{j}}\right), \quad j=1, \cdots, n,
$$

and thus establish (1). To do this let

$$
y_{j}=\sigma\left(a, b, x_{j}\right), \quad j=0, \cdots, n .
$$

Then

$$
y_{0}=\sigma\left(a, b, x_{0}\right)=\sigma(a, b, a)=a
$$

and

$$
y_{n}=\sigma\left(a, b, x_{n}\right)=\sigma(a, b, b)=b \text {. }
$$

Also

$$
y_{j-1}=\sigma\left(a, b, x_{j-1}\right) \underset{\theta_{i_{j}}}{\equiv} \sigma\left(a, b, x_{j}\right)=y_{j},
$$

since $x_{j-1} \equiv x_{j}\left(\theta_{i_{j}}\right)$ and $\theta_{i_{j}}$ has the substitution property. Finally, since $a \equiv b(\theta)$, we have

$$
\begin{aligned}
y_{j-1} & =\sigma\left(a, b, x_{j-1}\right) \underset{\theta}{\equiv} \sigma\left(a, a, x_{j-1}\right)=a \underset{\theta}{\equiv} b=\sigma\left(b, b, x_{j}\right) \\
& \equiv \sigma\left(a, b, x_{j}\right)=y_{j} .
\end{aligned}
$$

Hence (2) is established and with it (1). This proves the first statement of the theorem. 
To prove the second statement we need only show, by virtue of the first statement and Theorem 1, that (ii) implies (i) when $U$ is a permutable set of identities. Suppose, then, that $U$ is a permutable and distributive set of identities and construct $\mathfrak{F}_{3}(U)$ using the letters $a, b, c$ as generators. Observe that, in consequence of the fact that $\mathfrak{F}_{3}(U) \in C(U)$, we have $\theta_{1} \cup \theta_{2}=\theta_{1} \circ \theta_{2}$ and $\theta_{1} \cap\left(\theta_{2} \cup \theta_{3}\right)=\left(\theta_{1} \cap \theta_{2}\right)$ $\cup\left(\theta_{1} \cap \theta_{3}\right)$ (and dually) for all congruence relations $\theta_{1}, \theta_{2}, \theta_{3}$ on $\mathfrak{F}_{3}(U)$.

Next observe that identification of the generators $a$ and $b$ induces a congruence relation $\theta_{1}$ on $\mathfrak{F}_{3}(U)$. Similarly, identification of $a$ and $c$ induces $\theta_{2}$ and identification of $b$ and $c$ induces $\theta_{3}$ on $\mathfrak{F}_{3}(U)$. Hence we obtain congruence relations $\theta_{1}, \theta_{2}, \theta_{3}$ on $\mathfrak{F}_{3}(U)$ such that

$$
a \equiv b\left(\theta_{1}\right) \text { and } a \equiv b\left(\theta_{2} \circ \theta_{3}\right) \text {; }
$$

that is

$$
a \equiv b\left[\theta_{1} \cap\left(\theta_{2} \circ \theta_{3}\right)\right]
$$

Thus we have

$$
a \equiv b\left[\left(\theta_{1} \cap \theta_{2}\right) \circ\left(\theta_{1} \cap \theta_{3}\right)\right]
$$

and hence there is an $S$-expression $\sigma(a, b, c) \in \mathfrak{F}_{3}(U)$ such that

$$
\begin{aligned}
& a \equiv \sigma(a, b, c)\left(\theta_{1} \cap \theta_{2}\right), \\
& b \equiv \sigma(a, b, c)\left(\theta_{1} \cap \theta_{3}\right) .
\end{aligned}
$$

Hence

$$
\begin{aligned}
& a \equiv \sigma(a, a, c)\left(\theta_{1}\right), \\
& a \equiv \sigma(a, b, a)\left(\theta_{2}\right), \\
& b \equiv \sigma(a, b, b)\left(\theta_{3}\right) .
\end{aligned}
$$

But each of $\mathfrak{F}_{3}(U) / \theta_{1}, \mathfrak{F}_{3}(U) / \theta_{2}, \mathfrak{F}_{3}(U) / \theta_{3}$ is isomorphic with $\mathfrak{F}_{2}(U)$. It follows from the definition of $\mathfrak{F}_{2}(U)$ that each of

$$
\begin{aligned}
& \sigma(x, x, z)=x, \\
& \sigma(x, z, x)=x, \\
& \sigma(x, z, z)=z,
\end{aligned}
$$

is a consequence of the identities $U$. This completes the proof.

As an example consider the equational class of all lattices. Taking

$$
\sigma(x, y, z)=[(x \cap y) \cup z] \cap(x \cup y)
$$

it is easily checked that (i) of Theorem 2 is satisfied. This re-establishes the fact that lattices have distributive lattices of congruence 
relations (first established by Funayama and Nakayama [4] by making implicit use of the expression $\left.\left[(x \cap y) \cup_{z}\right] \cap(x \cup y)\right)$. If one considers the equational class of Boolean algebras with operations $\cup, \cap$, and ' (complementation), then, taking

$$
\pi(x, y, z)=\left[\left(x \cap y^{\prime}\right) \cup z\right] \cap\left(x \cup y^{\prime}\right)=\sigma\left(x, y^{\prime}, z\right),
$$

one has the conclusions of Theorem 2 for Boolean algebras.

3. Remarks. Theorem 2 provides an equational characterization of distributivity only in the presence of permutability. By an extension of the above argument one can also show that distributivity is characterizable by equations when, associated with the identities $U$, there is an integer $N$ such that for all congruence relations $\theta_{1}, \theta_{2}$ on $\mathscr{A} \in C(U)$, $\theta_{1} \cup \theta_{2}$ is a relative product $\theta_{1} \circ \theta_{2} \circ \theta_{1} \circ \cdots$ of length at most $N$. Moreover, it is not hard to show that for each $N$ this last condition is equationally characterizable; Theorem 1 does this for $N=2$. Whether this condition can be dropped or replaced by alternative conditions, which may or may not be equationally characterizable, remains an open question.

\section{BibLIOGRAPHY} 3-20.

1. A. Malcev, On the general theory of algebraic systems, Mat. Sb. (77) 35 (1954),

2. G. Birkhoff, Qn the structure of abstract algebras, Proc. Cambridge Philos. Soc. 31 (1935), 433-454.

3. - Lattice theory, Amer. Math. Soc. Collog. Publ. Vol. 25, rev. ed., Amer. Math. Soc., Providence, R. I., 1948.

4. N. Funayama and T. Nakayama, On the distributivity of a lattice of lattice congruences, Proc. Imp. Acad. Tokyo 18 (1942), 553-554.

San Francisco State College 